\title{
La inmanencia spinozista como forma de crítica política. Una reflexión desde el caso chileno
}

\section{Spinoza's Immanence as a Form of Political Criticism. A Reflection Based on the Chilean Situation}

\author{
Cristian Andrés Tejeda Gómez ${ }^{1}$ \\ Universidad de Los Lagos (Chile)
}

Recibido: 26-09-16

Aprobado: 25-06-17

\section{Resumen}

El concepto de inmanencia acuñado por Baruch Spinoza tiene la cualidad de expurgar todo contenido trascendente de su acepción. La filosofía de Spinoza es inmanente porque su sentido puede leerse a partir de una concepción horizontal del Ser, Dios o la Naturaleza. Del mismo modo, su filosofía política puede entenderse como una crítica a todas a las ilusiones y las vagas ideas del discurso político y también como una crítica de la democracia y el neoliberalismo. La mejor manera de exponer esta idea es a partir del examen de un caso ejemplar: Chile.

Palabras-clave: inmanencia, política, trascendencia, democracia, concentración de poder, ampliación de poder.

\footnotetext{
${ }^{1}$ (cristian.tejeda@ulagos.cl). Académico del Departamento de Educación de la Universidad de Los Lagos (Chile) e integrante del Núcleo de Investigación en Lengua, Educación y Discurso (NU 05/17). Periodista de la Universidad Austral y Licenciado en Comunicación Social de la Universidad Austral de Chile (2002); Licenciado en Filosofia de la Universidad de Chile (2008); Postítulo en Filosofía y Educación de la Universidad de Chile (2008-2009); Master (2010-2011) y Doctor en Ciudadanía y Derechos Humanos por la Universidad de Barcelona (2011-2015). Especialista en la filosofía de Baruch Spinoza, su tesis de doctorado se titula "Política y pasiones desde una perspectiva spinozista. La inmanencia en la filosofía de Spinoza y su potencia como herramienta político-afectiva". Sus líneas de investigación son filosofía política; filosofía moderna y contemporánea; y ciudadanía y educación.

Ha publicado en distintas revistas sobre el pensamiento de Spinoza (Revista de filosofía de Chile; Astrolabio. Revista internacional de filosofía y OXÍMORA Revista Internacional de Ética y Política de España) y también ha asistido a Congresos, Coloquios y seminarios de educación y filosofía a nivel nacional e internacional.
} 


\begin{abstract}
One of the characteristics of the concept of immanence, coined by Baruch Spinoza, is its expurgation of any content related to transcendence from its definition. Spinoza's philosophy is immanent because it is understood from the idea that Being, God, or Nature are at the same level. Similarly, his political philosophy can be understood as a form of criticism against all the illusions and vague ideas of political discourse, and also against democracy and neoliberalism. The best way to explain this idea is to put it in a real context: Chile.
\end{abstract}

Key-words: Immanence, Politics, Trascendence, Democracy, Power Concentration, Power Extension.

\title{
Introducción
}

La filosofía de Spinoza podría definirse como un pensamiento de la inmanencia y no exageramos al decir que si no llegara a estimarse como su concepto más importante, al menos es aquel que articula toda su filosofía de la forma más coherente posible (Yovel, 1995). Que la inmanencia es fundamental en Spinoza, es una tesis sostenida por uno de sus más prolijos estudiosos: Martial Gueroult (1968). Cuando en la prop. XVIII del libro I de la Ética Spinoza nos dice que "Dios, es causa inmanente, pero no transitiva de todas las cosas" (Spinoza, 2011: I, prop. XVIII, n. 85) no hace otra cosa que poner al Creador al interior de la creación y con ello admitir que tanto Él como las cosas tienen la misma calidad ontológica. Esto supone una equiparación de los conceptos de Dios y Naturaleza (Gebhardt, 2008), y una revalorización del cuerpo.

Yirmiyahu Yovel (1995) define la inmanencia desde su estudio de la obra de Spinoza como el principio que considera "este mundo" como único ser actual, la única fuente de valor y de la autoridad política. De este achatamiento ontológico que introduce la inmanencia, el cual recusa el desdoblamiento platónico o cartesiano del mundo (Zourabichvilli, 2014), se desprenden una serie de características. 1) No hay principios exteriores que expliquen el mundo, por lo mismo, Dios y todas las cosas se encuentran en la Naturaleza; 2) por añadidura, todo principio que dé explicación del orden de los sucesos es interior, lo que supone una horizontalidad de todo lo creado; 3 ) así, toda explicación solo puede ser pensada desde este mundo y no desde uno trascendente, lo que supone eliminar todo tipo de jerarquías como criterios explicativos de los procesos constituyentes de las cosas (Tejeda, 2015). 
$\mathrm{Si}$, para no confundirnos, obviamos la nomenclatura medieval-moderna de Spinoza y tratamos de dar una explicación de los sucesos contemporáneos mediante una teoría inmanente spinozista, deberíamos aplicar estos tres rasgos a la explicación de fenómenos visibles. Nos proponemos mostrar que la ilusión de la explicación trascendente, es decir, aquella que erige criterios jerárquicos y exteriores, está enraizada en nuestras propias visiones de mundo y prácticas. Es lo que se denomina habitualmente como naturalizar (Bourdieu y Passeron, 1996) o interpretando a Spinoza: el acto por el cual uno piensa como constituyente aquello que solo es constituido.

Una crítica inmanente de la política y la democracia es un ejercicio adecuado, más cuando proponemos centrarnos en la construcción de un capítulo particular de la historia: el Chile constituido durante la dictadura de Augusto Pinochet, la postdictadura y del desarrollo de políticas neoliberales. La selección del caso chileno no es antojadiza, pues tal como señala Naomi Klein (2007) Chile es la expresión de la primera contrarrevolución neoliberal en pos de la recuperación de los privilegios de clase arrebatados a las clases dirigentes en el periodo del Estado de Bienestar para Europa (Capella, 2007; Hobsbawm, 1994) o desarrollismo para Chile y Latinoamérica (Del pozo, 2009; Salazar y Pinto, 1999a). La implementación de medidas neoliberales bajo dictadura supuso la implementación de medidas extremas (Delano y Translaviña, 1989) las cuales introdujeron profundos cambios materiales y culturales en la sociedad chilena. En ese sentido, Chile es un barómetro comparativo clave para medir la profundidad de los cambios que hoy suceden en sociedades como España, Grecia o Irlanda, donde la tendencia a la "concentración de poder" neoliberal parece posicionarse como constitutiva del panorama político. Por lo mismo, justificamos la elección de Chile como elemento de análisis, pero sostenemos que la nomenclatura teórica que extraemos de la inmanencia spinozista, perfectamente puede ser replicada a una relectura de la situación de los anteriores países mencionados. En especial, los conceptos de "ampliación de poder" y "concentración de poder" que acuñamos siguiendo una interpretación propia del Tratado Político de Spinoza.

\section{La política inmanente spinozista}

La política inmanente spinozista encuentra su forma más acabada en la última obra de Spinoza: el Tratado Político. Podría esto expresar un contrasentido ya que aquel escrito quedo inconcluso y tiene evidentes desperfectos ${ }^{2}$ (Negri, 1993). La causa de aquello es la muerte de Spinoza, pero

\footnotetext{
${ }^{2}$ Toni Negri (1993) da un listado de esas dificultades en la Anomalia salvaje. Ensayo sobre poder y potencia en Baruch Spinoza.
}

Araucaria. Revista Iberoamericana de Filosofía, Politica y Humanidades, año 20, n 39 . Primer semestre de 2018. Pp. 187-209. ISSN 1575-6823 e-ISSN 2340-2199 doi: 10.12795/araucaria.2018.i39.09 
entendemos igualmente que el pensamiento maduro del filósofo está cobijado en sus últimas obras. El Tratado Político consta de dos partes claramente establecidas: del capítulo I al V se desarrollan los principios constituyentes de la política. Del capítulo VI hasta el XI, los productos de esos principios que no son otros que las formas políticas clásicas: monarquía, aristocracia y democracia (Balibar, 2011).

De los principios constituyentes, dos son los más relevantes: la potencia y la multitudo. El poder como proceso (potentia) es un elemento constituyente de la política y supone que sus límites no están dados por ninguna forma particular. Tal como en la Naturaleza spinozista completa ${ }^{3}$, la política solo es un espacio de despliegue del poder de los cuerpos hasta donde puedan perseverar. En Spinoza hay un anaformismo donde el despliegue de fuerzas en ejercicio es lo fundamental tanto a nivel cosmológico como político. El poder como instituido (potestas), es decir, el resultado de las formas de institucionalización corresponde a los productos de la política. Muy bien lo expresa Gilles Deleuze cuando dice que en Spinoza "la potencia es idéntica a la esencia misma" (1999: n. 82) y, por lo mismo, es lo que determina la constitución de las formas de organización política. No hay forma antes de la potencia constitutiva.

La conclusión tentativa que podríamos dar es que para Spinoza tanto la monarquía como la aristocracia y la democracia, no son más que formas instituidas y ninguna es superior ontológicamente a la otra. Pero aún parece pobre esta aseveración ya que Spinoza, debido a su muerte, no nos dice casi nada sobre la democracia en el Tratado político: solo unas cuantas ideas donde se establece el sentido general de la democracia, pero su desarrollo está inconcluso. ¿Cuál es el momento en qué señala esta paridad de las formas de gobierno, más aún cuando en el Tratado Teológico-Político (su obra política anterior) manifiesta con claridad una propensión a la democracia como forma privilegiada de gobierno? Se lee en esa obra:

\begin{abstract}
Existe, por tanto, la posibilidad de formar una sociedad sin contradecir el derecho natural y de mantener siempre con suma lealtad el pacto. La condición es que el individuo transfiera todo el poder que posee a la sociedad, que retendrá en exclusiva el máximo derecho natural sobre todas las cosas, es decir, el poder soberano, al que todos se verán obligados a obedecer libremente o por miedo al suplicio. Este derecho de la sociedad se llama democracia (Spinoza, 2014: cap. XVI, n. 242).
\end{abstract}

${ }^{3}$ Deleuze dirá que Dios o la Naturaleza "va a ser el concepto en tanto que reúne el conjunto de todas sus posibilidades" (Deleuze, 2008: n .23). En otros términos, es imposible de ser determinado, solo determina. Además, Spinoza refiriéndose a la Naturaleza completa en la Ética (Libro I, prop. XXIX, esc.) hará una distinción que refrenda nuestra diferenciación entre leyes constituyentes de la política y sus productos: por un lado, la Naturaleza naturante o leyes de constitución generales de las cosas; por otro lado, la Naturaleza naturada o todo lo que se sigue como producto de la posibilidad infinita de producción de la Naturaleza. 
Decíamos que el Tratado Político adolece de rigurosidad por la falta de revisión de su autor que fallece durante la redacción, pero hay un momento al interior de la segunda parte del Tratado Político (cap. VI a XI) donde Spinoza hace una afirmación de sumo interés: "La multitud puede mantener bajo el rey una libertad suficientemente amplia [satis amplam libertatem], con tal que logre que el poder [potentia] del rey se determine por el solo poder de la misma multitud y se mantenga con su solo apoyo. Y ésta ha sido la única regla [Regula] que yo he seguido al establecer las bases del Estado monárquico"4 (Spinoza, 2010: cap. VII, § XXXI, n. 179).

Este párrafo corresponde a la conclusión del capítulo VII sobre la forma de gobierno llamado monarquía. Lo relevante de esta afirmación solo puede ser captado en el idioma original de escritura. El matiz del latín nos da dos sentidos para un elemento que en nuestro idioma solo traduce uno: poder. Spinoza no hace referencia a la potestas, es decir, al poder institucionalizado, formado o realizado, sino a la potentia o poder determinante. Así, existe una regla: para que la multitudo (cuya potencia define al Estado5) mantenga "una libertad suficientemente amplia" es necesario que ella determine (pues la multitud no es determinada) la potencia del monarca. Además, esa Regula es la "única" que ha seguido para establecer las bases de la monarquía.

La única condición de la calidad de la monarquía es que sea lo más "amplia" (amplam) posible y esto es un principio constituyente de la política spinozista. Para los capítulos sobre la aristocracia y la democracia no existe conclusión formal del filósofo, pero podríamos afirmar que al ser una Regula fundada en la potentia, debe necesariamente aplicarse esta aseveración a las otras formas de gobierno. La consistencia de esta interpretación se aprecia si atendemos a la consideración del concepto de inmanencia: no puede haber causa exterior, todas las causas son interiores y deben atender a describir "este" mundo. Si la democracia fuera el mejor tipo de gobierno, tendría una jerarquía superior como forma de gobierno, daría explicación de los otros tipos de gobierno y se entendería como causa exterior de lo político. La pregunta es por qué se revela esto solo hasta el cap. VII y no del cap. I al V donde realmente se describen las leyes constituyentes de la política. La respuesta es que la muerte de Spinoza le impidió corregir muchos de los desperfectos del Tratado Político.

\footnotetext{
4 "Multitudinem satis amplam libertatem sub Rege servare posse, modò efficiat, ut Regis potentia solâ ipsius multitudinis potentiâ determinetur, \& ipsius multitudinis praesidio servetur. Atque haec unica fuit Regula, quam in jaciendis imperii Monarchici fundamentis sequutus sum" (Spinoza, 1972: cap. VII, § XXXI, n. 323).

5 "Este derecho que se define por el poder de la multitud suele denominarse Estado" (Spinoza, 2010: cap. II, § 17, n. 99).
} 
Deberíamos hacer otra consideración sobre el concepto de potencia. Si ella no puede ser determinada es porque su cualidad es ser continua y efectuarse a cada momento. A nivel de la naturaleza general Spinoza dirá en la Ética: "La potencia de Dios es su esencia misma" (Spinoza, 2011: prop. XXXIV, n. 107). Por lo mismo, toda la Naturaleza puede ser concebida como producción en acto. Al nivel de la política esto tiene una consecuencia particular: los productos de la política pueden ser considerados como el resultado de los encuentros dinámicos de todos los cuerpos sociales ${ }^{6}$. ¿Pero quién instituye esa dinámica de constitución? Precisamente el otro elemento constituyente al cual solo hemos hecho referencia: la multitudo. ¿Y cómo? A partir de una regla simple que se extrae del Tratado Político: mediante un "tendencia" hacia la "ampliación de poder" (potentia). Sin embargo, Spinoza igualmente aseverará en el Tratado Político que una cosa es gobernar y otra hacerlo muy bien (Spinoza, 2010: cap. $\mathrm{V}, \S 1)$. Su preocupación en el escrito es describir como pueda erigirse un buen gobierno, de lo cual se desprende implícitamente que hay causas por las cuales un gobierno puede tender hacia una dirección contraria: la "concentración de poder". Esto puede corroborarse fácticamente, pues distintas democracias (p.e., Estados Unidos, Venezuela o Chile) pueden describirse al tenor de sus diversas tendencias de "ampliación" o "concentración de poder".

Si Spinoza habla de multitudo y no de pueblo, no es por un simple afán terminológico (Virno, 2003). El filósofo entiende que los conceptos de Estado o individuo no sirven para explicar la dinámica política que se sigue de la noción de potencia como acto. Esta es una diferencia importante del Tratado Teológico-Político con respecto al Tratado Político. En esta última obra Spinoza pone a la multitudo como realidad intermedia entre dos términos extremos: "El problema político no es más un problema planteado en dos términos, sino en tres: «individuo» y «Estado» son en realidad abstracciones, que no tienen sentido más que en relación la una con la otra; cada uno expresa en definitiva una modalidad bajo la cual se realiza la potencia de la multitud como tal" (Balibar, 2011: n. 84). El individuo como proceso de conformación

\footnotetext{
${ }^{6}$ En un interesante artículo Luciano Rubio, al hablar de la política spinozista, introduce el concepto de ecosistema la que pone de manifiesto la importancia de la noción de dinamismo, potencia y cuerpo en la filosofía de Spinoza: "el término ecosistema significa aquí un conjunto articulado de partes en interacción regido por constancias internas y por un intercambio esencial con el medio, de manera que se trata de un sistema abierto en el cual el adentro y el afuera son rigurosamente recíprocos" (Espinoza, 2014). No hay política sin cuerpos ejerciendo activamente su potencia sobre otros cuerpos.

${ }^{7}$ El concepto de tendencia es definido por Henri Bergson. Pensamos que existe una afinidad notable entre las obras de los dos autores y por lo cual este concepto se acopla fácilmente a la nomenclatura spinozista: "No existen cosas hechas, sino solamente cosas que se hacen, no hay estados que se mantienen, sino solamente estados que cambian. El reposo nunca es más que aparente, o más bien relativo. La conciencia que tenemos de nuestra propia persona, en su continuo fluir, nos introduce en el interior de una realidad según el modelo de la cual debemos representarnos las demás. Toda realidad es por tanto tendencia, si se acepta llamar tendencia a un cambio de dirección en un estado naciente". (Bergson, 2013: n. 211)
} 
siempre es el resultado de encuentros con otros cuerpos y, por lo mismo, siempre hay grupos o multitudes que influye en su conformación. Pero tampoco puede totalizarse el vínculo de pertenencia de un grupo con el Estado, pues él jamás es capaz de subsumir o entablar un acuerdo total entre los deseos de las distintas multitudes y como señala Spinoza, sería una mistificación ignorar las pasiones y afectos de los hombres en la constitución de la política (Spinoza, 2010, cap. I §1). Y eso es precisamente lo que pasa con el concepto de pueblo: vuelve homogéneo, abstracto, aquello que inmanentemente se aparece como el resultado de multitudes heterogéneas. Más importante que el resultado es siempre la forma en que la potencia dinámica de los cuerpos se expresa y, por eso, la multitud como elemento constituyente no se determina $a$ priori.

En fin, lo fundamental de cualquier forma de gobierno en la política spinozista es el despliegue de la potencia que instituye la multitud y de acuerdo a ello podemos juzgar inmanentemente la "bondad" de una monarquía, democracia o aristocracia. Señala muy bien Balibar en relación al concepto de derecho que se extrae del Tratado Político que no es primero: "la noción primera es la de "poder". Se puede decir que la palabra "derecho" (Jus) expresa la realidad originaria del poder (potentia) en el lenguaje político" (Balibar, 2011: n. 74-75). Las tres formas de gobierno pueden funcionar o no hacerlo y eso es demostrable fácticamente, acudiendo a la descripción de las fuerzas de "este" mundo. La democracia no es ni la mejor forma de gobierno ni la ideal: solo es el Estado absoluto (Spinoza, cap. 2010, XI, §1). Pero en su constitución un Estado puede tender hacia dos direcciones: generar una "ampliación de poder" a toda la multitudo o una "concentración de poder"8 en solo una parte de la multitud. Estos dos conceptos que hemos acuñado a partir del Tratado Político - pues Spinoza no habla de estos dos términos - se justifican por la potencia como principio de la filosofía spinozista y serán de suma importancia para el examen de Chile como caso posible de examen.

\section{Chile y el análisis inmanente de la democracia y el neoliberalismo chileno}

Decíamos que la definición de inmanencia poseía tres caracteres. Si podemos traducirlos en términos políticos y en una revisión del concepto de democracia chileno y del neoliberalismo instaurado, podríamos señalar: 1) la democracia no puede ser erigida como la mejor forma de gobierno a priori (trascendencia) y, si viene decirlo al caso, es por la facilidad con que los gobiernos de distinta índole se autodenominan democracias para legitimar sus acciones; 2) toda democracia o sistema económico-político instaurado debe ser juzgada no por una regla

\footnotetext{
${ }^{8}$ La "ampliación" y la "concentración de poder" no solamente refieren a la economía, sino a todas las dimensiones en que se puede manifestar la vida.
}

Araucaria. Revista Iberoamericana de Filosofia, Política y Humanidades, año 20, n 39. Primer semestre de 2018 Pp. 187-209. ISSN 1575-6823 e-ISSN 2340-2199 doi: 10.12795/araucaria.2018.i39.09 
normativa o ideal democracia propuesta (jerarquía), sino por las causas interiores que desembocan en su particular constitución; 3) así, todas las explicaciones posibles solo pueden ser referidas a los encuentros sucedidos en tal o cual democracia o sistema económico-político de tal o cual país.

El caso chileno es relevante por ser el primer país en que el neoliberalismo se instauró bajo la forma de una revolución o contrarrevolución capitalista (Moulian, 2002). Al respecto David Harvey señala:

\begin{abstract}
Merece la pena recordar que el primer experimento de formación de un Estado neoliberal se produjo en Chile tras el golpe de Pinochet el «11 de septiembre menor» de 1973 (casi treinta años antes del día del anuncio del régimen que iba a instalarse en Iraq por parte de Bremer). El golpe contra el gobierno democráticamente elegido de Salvador Allende fue promovido por las elites económicas domésticas que se sentían amenazadas por el rumbo hacia el socialismo de su presidente. Contó con el respaldo de compañías estadounidenses, de la CIA, y del secretario de Estado estadounidense Henry Kissinger. Reprimió de manera violenta todos los movimientos sociales y las organizaciones políticas de izquierda y desmanteló todas las formas de organización popular (como los centros de salud comunitarios de los barrios pobres) que existían en el país. El mercado de trabajo, a su vez, fue «liberado» de las restricciones reglamentarias o institucionales (el poder de los sindicatos, por ejemplo). ¿Pero de qué modo iba a ser reactivada su estancada economía? Las políticas de sustitución de las importaciones (fomentando las industrias nacionales mediante subvenciones o medidas de protección arancelaria) que habían dominado las tentativas latinoamericanas de desarrollo económico, habían caído en el descrédito, particularmente en Chile, donde nunca habían funcionado especialmente bien (2007: n. 14).
\end{abstract}

Esta cita contextualiza la importancia mundial del caso chileno desde una perspectiva genealógica. Pero hay muchas particularidades en la forma de instaurar la democracia y el neoliberalismo chileno y es lo que le da ese carácter tan fuertemente extremista (Salazar, 2012). El 11 de septiembre de 1980 y con un plebiscito fraudulento, se aprueba la Constitución Política de la República de Chile de 1980. "La dictadura decidió usar el plebiscito pero sin aceptar, en realidad, someterse a sus resultados. Por ello se montó la consulta popular en condiciones tales que hacía imposible cualquier control efectivo de ella por parte de los opositores" (Moulian, 2002: n. 235). Ante tal situación, la oposición no solo fue interna, sino que en el mundo entero se condenó la falta de legitimidad del proceso (Cristi y Ruiz-Tagle, 2006). El artículo $4^{\circ}$ de la Constitución afirma brevemente "Chile es una república democrática" (Constitución de Chile, 1980). Preguntarnos por la veracidad de esta afirmación durante los años 80 a 89 no tiene sentido. Nuestras preguntas son, ¿cómo se puede transitar exitosamente de una dictadura a una democracia manteniendo una constitución aprobada sin garantías de legitimidad? ¿Cuál es el sentido de 
la democracia chilena y el neoliberalismo? El 11 de marzo de 1990 se realizó la ceremonia de trasmisión de mando de Augusto Pinochet al presidente Patricio Alwyn Azócar de la Concertación de Partidos por la Democracia, la agrupación política que por mayor cantidad de años ha administrado la política estatal (la derecha política solo estuvo en el poder con el presidente Sebastián Piñera en el periodo 2010-2014) .

A partir de allí comienza el proceso llamado de "transición a la democracia" cuya fecha de finalización corresponde al periodo de cuatro años en que Patricio Alwyn cumplió sus funciones de presidente. Durante su mandato y con el retorno de la democracia se hacen una serie de reformas necesarias a la constitución. Sin embargo, que ese proceso de transición haya cumplido su ciclo es discutido. Algunos opinan que la transición ha acabado recién el 2005 con un paquete de reformas que Ricardo Lagos aprobó durante su mandato y que lo llevaron a afirmar delante de la prensa internacional "ahora podemos decir que la transición en Chile ha concluido" (BBC Mundo, 2005). Aquí habría que preguntarse si la transición acaba cuando se generan reformas constitucionales o cuando de facto se erige una nueva constitución (Atria, 2016). Otros, como el escritor Jorge Edwards, ganador del premio Cervantes en 1999, opina que "la transición no ha terminado y la reconciliación no ha comenzado" (El País, 2005). Si la transición no ha acabado, tiene sentido preguntarnos por el carácter de la democracia chilena, pero para eso tendríamos que demostrar que ella no ha concluido. Eso significaría que hay ciertas ataduras que no han permitido a Chile desvincularse de los derroteros de la dictadura militar y que, por lo mismo, el sentido de la democracia en que se vive es muy singular. Nosotros partimos de la afirmación de Renato Cristi y Pablo Ruiz-Tagle quienes aseveran que desde 1989 fue posible restaurar el constitucionalismo democrático chileno, pero solo parcialmente debido al "lento proceso de transición que todavía no ha terminado" (2006: n. 15-16). Para apoyar esta afirmación nos concentramos en el tipo y sentido de la democracia construida en Chile y lo que ella justifica: el orden económico.

\section{La democracia en transición}

El modelo que ha primado en Chile desde 1989 corresponde en grandes líneas a una democracia consensual, consociativa o elitista y eso es algo que escasamente se entrevé en los discursos políticos que siempre hablan de democracia a secas. En una bella frase Tomás Moulian nos señala que "el consenso es la etapa superior del olvido" (2002: n. 42) y lo que se borra de la memoria es que el consenso nunca es un dispositivo neutro. La interpretación

\footnotetext{
${ }^{9}$ Sin embargo, el 11 de marzo de 2018 Sebastián Piñera asume su segundo periodo de gobierno. 
que predominó en el periodo anterior al quiebre militar fue que polarización de tendencias políticas llevó a una necesaria inestabilidad en 1973 y a la intervención militar. La pérdida de consenso entre las partes tuvo como lección la necesidad de establecer lo que también se denominó "democracia de los acuerdos”. En 1985 el Centro de Estudios Públicos chileno (CEP) organizó un seminario para la discusión del texto Acuerdo Nacional para la Transición a la Plena Democracia, suscrito por un grupo de dirigentes políticos entre los que se contaba a Patricio Alwyn. En el seminario transcrito con el nombre Acuerdo Nacional y Transición a la Democracia el político chileno Edgardo Boeninger se adscribía plenamente a esta interpretación histórica de lo sucedido en Chile. La democracia consociativa es un aporte al futuro orden democrático, pues el acuerdo nacional permite reunir "a opositores al régimen militar y a sectores que han sido sus partidarios y que hoy en día están en una actitud independiente, tendiéndose así, también por primera vez, un puente que tal vez ayude a superar la polarización imperante" (Molina, Zabala, Avetikián, Flisfisch, Godoy y Guzmán, 1986: n. 7).

Para Carlos Ruiz Schneider, sin embargo, estas interpretaciones sobre las causas de lo sucedido tras el quiebre institucional chileno, son demasiado reduccionistas. Olvidan elementos fundamentales: "Mientras, por una parte, tienden a marginalizar el rol de la enormes desigualdades sociales en la gestación de los conflictos, sobre enfatizan por otra parte los efectos de la polarización «política» e ideológica, y del régimen institucional en el fin de la democracia en Chile" (Ruiz, 1993: n. 168). Es importante subrayar esto, pues se ha forjado un cierto diseño de modelo democrático que fue implementado a partir de una interpretación vencedora: en 1973 en Chile la polarización volvió completamente inestable el sistema político. Conclusión: se necesita una fórmula que estabilice las posiciones políticas. Siendo o no adecuada esta mirada, los efectos de su implementación propendieron a un cambio radical en el modo de hacer política y, por lo mismo, de comprender la democracia. Ruiz Schneider cita a uno de los primeros expositores chilenos de este tipo de modelos, Alberto van Klaveren y de él se extraen algunas consecuencias. Cito:

El modelo de la democracia consociativa postula que las sociedades fuertemente fragmentadas desde un punto de vista político pueden ser estabilizadas a través de un esfuerzo consciente de sus élites políticas, orientado a la búsqueda deliberada de medios que permitan contrarrestar los efectos centrífugos de esa fragmentación... La clave para entender esta paradoja parece estar en el comportamiento de las élites. Para poder neutralizar las divisiones políticas, ellas deben abandonar las prácticas competitivas y el principio de las mayorías simples... y formar en cambio grandes coaliciones que incluyan a los principales grupos políticos existentes en la sociedad... En el fondo el consociativismo implica la idea de una tregua más o menos permanente entre bloques políticos rigurosamente distintos que, si bien se oponen unos a otros, se ven obligados 
a tolerarse y aceptarse mutuamente como realidades básicas que no pueden ni deben cambiarse (Ruiz, 1993: n. 169-170).

Primera consideración para un análisis inmanente. La democracia chilena, aún hoy, descansa en una teoría elitista del poder político, la que "está sobre todo marcada por su desconfianza en la participación política amplia" (Ruiz, 1993: n. 168). El papel central en el juego político es asumido por la clase política quienes son capaces realmente de tener un modelo estable de democracia y no ya la multitud. Es decir, se acepta solo un sentido de democracia como el verdadero, se erige como única norma posible (principio exterior que rige lo que debe ser considerado como democracia) y se establece que en ella solo las élites del poder pueden ser las conductoras (la única conducción posible corresponde a una jerarquía establecida). De esta forma, el límite puesto al poder de las minorías dirigentes es borrado y lo que se socava es la participación social en los procesos de construcción política de la mayoría. Si la democracia, como la define Spinoza, es la ampliación de la potentia a la mayor parte del cuerpo social que es la multitudo (Spinoza, 2010: cap. XI, § I, n. 243), la circunstancia chilena parece estar definida mediante un oxímoron. ¿Puede una democracia ser cautelada por una minoría y a la vez responder al criterio de una mayoría? No es necesario ni pertinente acudir a una definición; es posible recurrir a criterios inmanentes para describir el tipo de democracia que Chile ha forjado.

Si tomamos la frase de Boeninguer "se ven obligados a tolerarse y aceptarse mutuamente como realidades básicas que no pueden ni deben cambiarse", parece entenderse que a pesar de la oposiciones normales de cualquier sociedad y sus clases, tales grupos deben entenderse como realidades necesarias y naturales. Dice Bourdieu que "el olvido de la génesis que se expresa en la ilusión ingenua del «siempre-así», y también los usos substancialistas de la noción de inconsciente cultural, pueden conducir a eternizar y, más tarde, a «naturalizar» relaciones significantes que son producto de la historia" (Bourdieu, 1996: n. 49). Esa naturalización de la posición de los sectores sociales le otorga a los productos de la política, es decir, grupos con poder, la calidad de realidades constituyentes y eso es precisamente lo que rechaza Spinoza. Señala Ruiz que este modelo tiene más sentido "en sociedades con divisiones étnicas, culturales o religiosas" que en "sociedades como la chilena, en donde la división social fundamental arraiga en desigualdades socioeconómicas considerables" (Ruiz, 1993: n. 171). Con todo, las dos grandes agrupaciones políticas que permanecen hasta hoy en la alternancia del poder son la Concertación (hoy Nueva Mayoría) y la Alianza (hoy Chile Vamos).

La democracia de consensos también ha sido pensada por otros políticos chilenos como Alejandro Foxley. Él señala que el modelo construido busca reducir el peligro presente en la amenaza de corrientes ideológicas que no puedan conciliarse. Por eso, las democracias consociativas "optan por gobiernos 
que sobrerrepresentan a las minorías, antes que por una alternancia total en el poder con gobiernos de la mayoría, excluyentes de la minoría” (Ruiz, 1993: n. 171). Esta desconfianza por las élites podría pensarse como injustificada; sin embargo, la pregunta a responder es quienes son esas minorías que pactan el retorno a la democracia: militares, políticos y empresarios. Ya "los primeros dos años de la dictadura son de un consenso dentro de las clases dominantes de restaurar los pilares de la acumulación" (Mayol y Ahumada, 2015: n. 90) o de asegurar la "concentración de poder" a favor de estas minorías. A esto es lo que autores como Moulian (2002), Klein (2007), Capella (2007) o Harvey (2007) se refieren cuando hablan de contrarrevolución a secas o contrarrevolución conservadora: y esta no es solo como un proceso local, sino también mundial. Por lo mismo, la supuesta neutralidad de la explicación histórica para una salida pactada en Chile es un discurso vago (o ilusorio), pues tiene como fondo inexorable la decisión de los vencedores.

Otro rasgo importante que se desprende de esta "elitización" de la democracia tiene que ver con lo que Foxley denomina "tema tecnocrático del combate contra las ideologías" (Ruiz, 1993: n, 171). La política es un tema de especialistas que dialogan sobre la manera de hacer más estable la democracia para llegar a los acuerdos necesarios, lo que conduce a la despolitización de las mayorías. Esto también coincide con la visión conservadora de que la política es la decisión tomada por una jerarquía de sabios que trasciende a la decisión común. Pero la efectividad de esta despolitización sistemática de la mayoría necesitaba de un proceso legitimador. Luego de las consecuencias de una sangrienta dictadura para la población chilena, existieron dos momentos de alzamiento popular durante la década de los 80' para derrocar la dictadura (Salazar y Pinto, 1999b). Se imponía la necesidad de un tránsito hacia la democracia, pues sería difícil la aceptación de este nuevo pacto sin extirpar el miedo. Así. "durante el gobierno de Patricio Aylwin (1990-1994) se legitima en democracia la política económica liberal" (Ruiz y Boccardo, 2014: n. 29) y el tejido social que combate por el objetivo del derrocamiento militar entra en un periodo de mitigación de su actividad. Es por lo mismo que declara Salazar: "el movimiento social entra al 2000 en condiciones distintas a la que ocurrió en el 1900. Las bases sociales han perdido protagonismo y sus organizaciones carecen de representatividad" (Salazar, 1999b: 131).

\section{Una democracia a la medida del sistema económico}

Lo que se desprende de esta explicación es mucho más importante y solo lo habíamos tocado tangencialmente: los efectos el sistema económico. Para hacer consecuente este modelo de democracia con las políticas neoliberales 
implementadas desde 1975, se concibe al mercado como un espacio neutro que funciona de acuerdo a las leyes de la mejor distribución para todos. "La opción por la economía de mercado se transforma así en una especie de principio metapolítico, que pone límites externos a un ejercicio consociativo o concertado de las políticas sectoriales" (Ruiz, 1993: n.176). Un principio metapolítico neutro como límite externo configurador, no es otra cosa que la erección de un principio transcendente. La democracia de acuerdos, por lo mismo, "es el reconocimiento sin ambages del derecho de propiedad privada, incluidos los medios de producción, y la postulación del mercado, junto a la concertación y la acción del Estado como los mecanismos más eficientes de asignación de recursos" (Ruiz, 1993: n. 182).

Es la constitución chilena de 1980 que hasta hoy se consagra, la que con más o menos modificaciones, "privilegia la representación por sobre la participación democrática y consagra constitucionalmente un sistema neoliberal o libertario, claramente anti-republicano" (Cristi y Ruiz Tagle, 2006: n.15). Y precisamente el consenso político es lo que hace a los conglomerados olvidar sus antiguas diferencias, pues no son precisamente los gobiernos de derecha los que han extremado las políticas neoliberales.

\begin{abstract}
El gobierno de Lagos [2000-2006] consolida el giro neoliberal con una apertura sin par en América Latina y un manejo macroeconómico que aumenta el peso de los grupos económicos exportadores y financieros, con lo cual el empresariado termina de ajustar su actitud de aceptación hacia la Concertación y deja atrás una relación casi orgánica con los partidos de derecha (Campero, 2010 cit. en: Ruiz, 2014: n. 33).
\end{abstract}

Los efectos de la "concentración de poder" a partir de la intocabilidad del principio de la propiedad privada, justificada por los conglomerados políticos, y sus efectos extremos puede constatarse en todos los niveles de la vida chilena. En 1979 se inicia "con el objetivo de extender los principios del modelo [neoliberal] de Chicago hacia otros planos de las relaciones sociales" (Délano y Translaviña, 1989: n. 74) las llamadas "modernizaciones" del Estado.

Mayol y Ahumada (2015) señalan justamente que siguiendo la huella de la idea de Modernización es cómo entenderemos la naturalización y el olvido de la constitución de las actuales relaciones sociales y económicas de distribución de poder:

Hasta hoy se ha impuesto entre unos y otros, entre la derecha y la izquierda, entre complacientes y críticos, una gran convicción: Chile se aproxima al desarrollo, Chile se ha modernizado. Es lo que llamamos la Hipótesis Modernizadora [...] La Hipótesis Modernizadora implica una consecuencia: cualquier decisión a tomar no debe sacarnos de la senda. Chile debe caminar por la actual ruta (n. 12). 
Esta idea modernizadora según Mayol y Ahumada es falsa y nosotros apoyamos esta hipótesis, pues se aprecia, como antes argumentamos, que la "tendencia" general es la "concentración de poder" tanto en términos del sistema político (democracia elitista o decisión de unos pocos) como el sistema económico (distribución de riquezas y blindaje de los criterios neoliberales). El costo de la modernización es elevado y se expresa al nivel de la vida cotidiana. Hubo "un amplio proceso de redistribución del ingreso de los sectores bajos y medios hacia los sectores ricos" (Mayol y Ahumada, 2015: n.115). Pero la extensión de esa Modernización general se expresa en términos concretos en las modernizaciones específicas y mencionaremos cuatro: Plan Laboral, Reforma Previsional, Reestructuración de la Salud, La Reestructuración del Sistema Educativo. Precisamente, los procesos iniciados a partir de estas modernizaciones tienden hacia lo confirmación de dos cosas: la expansión de la neoliberalización hacia todas las formas de vida y a la fragmentación del cuerpo social en favor de solo una parte de ella. Describiremos con mayor detalle la última (educación), por el peso mediático y de movilización social que tiene en los últimos años.

La Reforma laboral fue hecha para disminuir el poder de los sindicatos y para redistribuir la riqueza en manos privadas. "Las asignaciones familiares a pesar de la propaganda acerca de la igualdad del tratamiento para obreros y empleados, son reducidas al $70.0 \%$ de su valor real comparadas con aquellas de 1970" (Urrea, 2014: n. 258). La Reforma Previsional creó las llamadas Administradores de Fondos de Pensiones (AFP). Se pasa así de un sistema solidario para asegurar las previsiones luego de la jubilación a una gran privatización del dinero de los fondos de pensiones. "Cada trabajador cotiza obligatoriamente durante su vida activa. Los fondos que él reúne son administrados por las AFP, que son empresas de servicios con fines de lucro. Las AFP invierten los fondos de los trabajadores en distintas actividades e instrumentos financieros, con el objeto de hacerlos crecer en el tiempo" (Délano y Translaviña, 1989: n. 79). En resumen, se traspasan los fondos para que ellas hagan negocios y hagan subir su rentabilidad. Si se gana, todos ganan, pero especialmente quienes administran las AFP. En caso de pérdida, quienes asumen son los que cotizan. Se llega a extremos tan patéticos como el caso de un trabajador cuya pensión es de 200 (1/3 de dólar aproximadamente) pesos chilenos mensuales (La Nación, 2014). La Reestructuración de la Salud creó las Instituciones de Salud Previsional (Isapres) que se dedican al lucro y que también crean una brecha entre los que pueden y no pagar por su salud. "Las Isapres organizan su funcionamiento en base a contratos individuales que establecen los servicios a proveer a la persona afectada por alguna afección y/o sus cargas, los cuales irán hasta el monto establecido en los contratos y no más allá" (Urrea, 2014: n. 303). Esto no asegura la atención a las personas, pero "las 
personas rechazadas por las Isapres son acogidas por Fonasa" (Urrea, 2014: n. 304), sistema de atención público que se lleva la mayor carga en número de atención de pacientes, las personas con más riesgos a sufrir dolencias y, por supuesto, las más pobres. Estas son tres formas de despotenciación de la vida y que afectan al proceso de desarrollo de los individuos: las asignaciones son el acceso a mayores bienes de disfrute material; las pensiones refieren a la posibilidad de continuación de una vida luego de agotada sus condiciones de productividad; y la salud reporta la posibilidad de un retorno a las condiciones habituales de nuestro organismo cuando sucumbe por alguna enfermedad y eso puede ocurrir en cualquier etapa de la vida. Todas estas dimensiones toman en Chile un cariz marcadamente económico y solo una parte reducida de la población tiene acceso efectivo y completo a estas prerrogativas: aquel sector minoritario donde se concentra la mayor cantidad de poder.

Si describimos más ampliamente los resultados del proyecto neoliberal en educación, es porque existe una relación palpable entre la cantidad y la calidad de la educación entregada y la posibilidad de acceso a los bienes materiales y servicios básicos. Y precisamente, no es coincidencia que las modernizaciones neoliberales atacaran estratégicamente el alma desde donde las transformaciones sociales pueden generarse: el conocimiento. Según Carlos Ruiz son dos los objetivos que se buscó con las políticas educativas post-dictadura. El primero "tiene que ver con el proyecto neoliberal global del Estado democrático-social, lo que se expresa en una reducción drástica del gasto fiscal en educación y en general de todo el gasto social. El peso del sector educacional es muy importante, ya que su financiamiento representa aproximadamente un $20 \%$ del gasto fiscal total" (Ruiz, 2010: n. 103). El otro, en consonancia con las políticas de la etapa anterior, busca un cierto disciplinamiento de sus actores principales: docentes y estudiantes (en especial, universitarios). A los profesores se les busca asimilar a la lógica de mercado proyectándolos como empleados que venden un producto (conocimiento y habilidades) en el área privada para sus consumidores. A los estudiantes, por sobre todo universitarios, se apunta a desincentivar su actividad política "a través de la implementación de una racionalidad económica costo/ beneficio, cuya base es el término del carácter gratuito de la educación superior y, simultáneamente, el agravamiento de las sanciones para la actividad política" (Ruiz, 2010: n. 104). El primer paso para la implementación de estas directrices es el traspaso de las escuelas a las municipalidades. Con ello, se aprecia que el papel del Estado queda disminuido y solo aparece como un ente supervisor. "La municipalización no es en sí misma este desmantelamiento, pero el traspaso que emprende el régimen militar apunta ya a un punto central de ese desmantelamiento: a la transformación de los docentes en empleados del área privada" (Ruiz, 2010: n. 107). El salto viene dado por la implementación del sistema de subvenciones, en el cual pasa a tener un rol primordial la lógica 
de mercado. Un artículo del ingeniero comercial Gerardo Jofré, asesor del Ministerio de Hacienda de la época, resume las concepciones neoliberales acerca de la educación. Tiene valor examinarlo, pues en él se parte por una concepción ideal de la educación bajo las directrices de mercado, decantándose finalmente por la educación subvencionada que es aplicada desde 1980. Parte el documento señalando que "las familias desean educar a sus hijos y pueden, en principio, sufragar los costos de hacerlo con los mayores ingresos que la educación genera" (Jofré, 1988: n. 193). Desde un comienzo se reconoce, como la constitución lo señalara, que la familia debe y puede pagar por la educación, aunque se reconozca que las familias pobres no pueden. Igualmente al inicio del escrito se señala con énfasis que "la intervención estatal debe hacerse generando incentivos correctos e intensos. Para ello los subsidios deben aplicarse sólo en Educación General, y créditos a la Educación Superior. El financiamiento a los establecimientos debe ser en función del servicio prestado. Las escuelas deben transmitir los incentivos a los profesores" (Jofré, 1988: n. 193). Los incentivos se inscriben también en la lógica de la competencia propia del mercado. Para los profesores las remuneraciones deben depender del éxito o fracaso del establecimiento para conseguir los recursos, lo que estimula por cierto la eficiencia y dedicación de cada uno. Los empresarios que tienen la función de diversificar la propuesta educacional abriendo establecimientos para la demanda, se verán igualmente sometidos al escrutinio de la eficiencia, en especial, en torno a la calidad de la educación que entregan. El pensamiento de fondo que aquí se compromete es que el empresario no se arriesgará a entregar una educación que minimice la utilidad por maximizar las utilidades, pues la competencia de mejor rendimiento lo haría desaparecer. En último término, estos incentivos también se traspasan a los estudiantes mediante la lógica de la competencia (Ruiz, 2010).

Ruiz (2010) dedica algunas críticas a los fundamentos de estas ideas. Establece que "el primer gran supuesto es que el sistema de la libre enseñanza y la competencia por los estudiantes, que hace recomendable un sistema de subvenciones a la demanda, tenga como resultado necesario una educación de más calidad" (Ruiz, 2010: n. 115). Este supuesto implica satisfacer dos condiciones: que las familias se interesan efectivamente por la calidad académica de la educación (su libertad de elegir el mejor establecimiento); y que se entregue información oportuna y completa de la calidad de los establecimientos. Para Ruiz ninguna de estas dos condiciones se cumple debido a que la elección de los padres suele hacerse por criterios más prácticos: cercanía, grupo social de los alumnos, religión, prestigio social. Tampoco la información ha llegado a tiempo para padres que podrían interesarse para tomar decisiones racionales en razón de ella. Al contrario, y más interesante aún, son las secuelas. Primero, "el sistema de educación subvencionada implica fuertes exigencias respecto de 
los profesores, especialmente en lo que tiene que ver con las modalidades de su contratación y en general con su estatus laboral" (Ruiz, 2010: n. 116). Si el sueldo del profesor depende de la demanda del establecimiento, entonces los incentivos para que el profesor consiga objetivos consistirán en una carga extra para obtenerlos, pues siempre existe la posibilidad de ser reemplazados bajo el modelo de la oferta y la demanda. Segundo, "la principal garantía para que un establecimiento que depende de la demanda se mantenga en competencia, es que atraiga el mayor número posible de alumnos" (Ruiz, 2010: n. 116). Este criterio tiene poca relación con la calidad; es más, atenta contra ella cuando una sala de clases repleta, no permite al profesor llevar de la mejor forma la dirección de una clase. Pero lo tercero, y más importante de señalar, es que como la educación subvencionada o completamente privada es dificultoso que sea pagada por las clases más modestas, el sistema de educación pública acoge a todos ellos que no pueden hacerlo. El resultado "es un sistema diferenciado por ingreso de los padres, reproductor de las desigualdades económicas y de clase y fuertemente segmentado" (Ruiz, 2010: n. 118). Si a nivel general durante todo este análisis inmanente de Chile, constatamos el fraccionamiento o descuelgue evidente de un grupo minoritario por sobre la gran mayoría (lo que llamamos la democracia consociativa), vemos que al nivel de la vida práctica se reproduce este mismo esquema.

En definitiva, a nivel del cuerpo social se reproduce un similar quiebre en educación que el que produce la instauración general del modelo de neoliberalización extrema, democracia de consenso y las otras modernizaciones específicas. Uno de los factores que permite en Chile acceder a mejores remuneraciones y, por lo mismo, tener acceso a mejores servicios (salud, vivienda, previsión social), es la entrada a la Universidad. Por lo mismo, consideremos algunas cifras que son aclaratorias. La prueba de selección universitaria (PSU) para el 2011 revela que, sobre la totalidad del alumnado (248.651 estudiantes), mientras que el $40 \%$ asociado a la enseñanza municipal (en total 99.221 estudiantes) logra el $17.7 \%$ de los mejores puntajes nacionales, el $10.0 \%$ de los alumnos de enseñanza privada (25.152 estudiantes) obtiene el $65.8 \%$. Las colegios subvencionadas logran un $16.6 \%$ (de un total de 121.983 estudiantes) ${ }^{10}$. Si se releva mayor el porcentaje de los liceos públicos que el de los colegios subvencionados es porque tres liceos públicos de tradición(Instituto Nacional, Liceo Lastarria y Manuel Barros Borgoño) logran en conjunto 6.9\% de los mejores puntajes, siendo para el resto de los establecimientos públicos del país la cifra solo de 4.8. "Dos explicaciones acerca de esta asimetría: la diferencia de los medios económicos de los que disponen los colegios privados y los municipales y el origen y el contexto socioeconómico familiar de los

\footnotetext{
${ }^{10}$ La cifras de la prueba de selección universitaria (PSU) de 2017 para el ingreso 2018, no revela cambios significativos en esta tendencia
}

Araucaria. Revista Iberoamericana de Filosofía, Politica y Humanidades, año 20, n 39 . Primer semestre de 2018. Pp. 187-209. ISSN 1575-6823 e-ISSN 2340-2199 doi: 10.12795/araucaria.2018.i39.09 
estudiantes, el capital cultural" (Urrea, 2014: n. 389). Por lo mismo, estos datos no son más que el reflejo de la fractura del cuerpo social proyectada por las directrices político-económicas y reproducidas en la vida misma. En cierto sentido, no tienen nada de novedoso si atendemos a las investigaciones del sistema escolar que hacen Bourdieu y Passeron (1996) para el contexto francés, pero que tienen plena vigencia al cotejar los datos de la educación chilena. $\mathrm{Su}$ resultado son las desigualdades que se reproducen y la "concentración de poder" propia de una minoría que se ha desgajado de la mayoría social y aunque el contexto educativo reciente nos evidencie un cambio de la situación, se debe estar a la espera de cuáles serán los efectos de esas transformaciones. Sin embargo, muchas de estas conclusiones sobre la educación se encuentran plenamente reflejadas en el marco educativo actual.

Todo esto conforma un panorama donde la máxima spinozista que establece que la virtud del Estado es la seguridad (Spinoza, 2010), no se cumple. Precisamente porque el metaprincipio político sobre el cual se asienta la institucionalidad chilena genera malestar creciente y falta de legitimidad de la política. La propiedad privada dejada actuar libremente y sin regulaciones genera un quiebre entre una clase propietaria que se desgaja del cuerpo social y otra que, aunque admitiendo niveles de distinción en la apropiación, se lleva un trozo más pequeño del reparto de la riquezas y acceso a los servicios. La democracia y el neoliberalismo en Chile, por tanto, establecen una relación de simbiosis que genera una amplia "concentración de poder" en torno a todos los aspectos de la vida.

\section{Conclusiones}

En términos de la crítica spinozista inmanente diríamos que aquello que es fundamental en política se troca por su derivado: se da una respuesta sobre lo que es democracia antes de considerar la multitudo y su poder constituyente como problema. Es decir, antes de que el cuerpo social tome posición sobre el problema de su constitución, se suplanta su poder por una minoría que se desgaja de él, que decide sobre la forma y el contenido de la democracia y lo asume como la única respuesta posible. Respondiendo a una pregunta planteada en el desarrollo del texto decimos: una democracia no puede ser cautelada por una minoría y a la vez responder al criterio de la mayoría o al menos no en el caso chileno. La transición a la democracia no puede concluir arrastrando elementos que se forjaron durante dictadura, por ejemplo, su constitución política. Se eterniza el principio metapolítico económico que es el mercado basado en el poder de una clase minoritaria que toma las decisiones. "No estamos pues, en presencia de un puro acuerdo sobre las reglas del juego democrático, como 
suelo constitucional de la política y de la deliberación sobre fines, sino que, a la inversa, es porque se concuerda sobre fines, como la sociedad de mercado, que se acepta pactar sobre las reglas del juego democrático" (Ruiz, 1993: n. 190). Y permítasenos recordar quienes son los que concuerdan sobre los fines de este acuerdo que permite el funcionamiento de la democracia (y no al contrario, que la democracia permita el acuerdo sobre el fin): "en definitiva, la transición ha sido un producto de la negociación y la aceptación de un conjunto de arreglos o pactos que, principalmente, definieron las áreas vitales de interés para las elites (militares, políticos y empresarios). Estos acuerdos básicos entre las elites sobre las reglas del juego han llevado a una democracia limitada" (Cazor, 2000).

El análisis desde una perspectiva inmanente de la democracia chilena nos deja mucho más. En el Tratado Político, basados en la idea de "ampliación de poder" nos muestra que para nada existe una suerte de gobierno ideal superior a otros. Una cosa es gobernar y otra cosa es hacerlo bien y Spinoza piensa que eso puede hacerse tanto bajo un régimen monárquico, aristocrático o democrático. Aunque una teoría democrática no llegue a ser desarrollada a cabalidad por Spinoza debido a su muerte, deja claros indicios de que a diferencia del Tratado Teológico-Político, una teoría inmanente de la política no puede elevar a un metanivel una forma de gobierno particular. Eso es porque la esencia del problema político constituyente de la multitudo es la "ampliación" y la "concentración de poder" y Chile es el caso manifiesto de que las luchas históricas no coinciden con el establecimiento de la democracia como mejor forma de gobierno, el periodo que analizamos. Podría contraargumentársenos que aquella democracia chilena es falsa y que es posible pulir la idea para llegar al establecimiento de "la" verdadera democracia. Pero esa idea no toma en cuenta que el ejercicio de una teoría siempre tiene efectos y que no hay nada de falso en las consecuencias de la teoría democrática elitista concebida para Chile en el periodo postdictatorial. Chile, como la expresión de la primera contrarrevolución neoliberal en el siglo XX para recobrar los privilegios de clase, da muestras que la democracia, que muchos estados erigen como bandera de legitimación, ha mutado profundamente de significado.

La inmanencia nos muestra que la esencia del problema político se encuentra en el constante despliegue del ejercicio de su potencia. No coincide con un estado fijo ni tampoco con alguna solución ideal que se presente como un puerto de destino final. Después de todo, si existiera una mejor forma de gobierno a la que pudiéramos llegar e hipotéticamente la alcanzáramos, sería el fin de la política y de la historia (Balibar, 2011). Eso es imposible, porque el plano social es la constitución de múltiples transformaciones, encuentros y movimientos que jamás cesan. Sin embargo, a pesar de todo la transición a la democracia fue anhelada por todos los chilenos y a condición de soslayar la cruel dictadura se aceptó que la democracia era la condición sine qua non para 
el arribo de la alegría. Hasta hoy la idea de que hemos transitado sanamente desde una dictadura a una democracia es un discurso asumido por gran parte de la población chilena y la comunidad internacional. Y, sin embargo, los lastres de la dictadura como el pacto entre militares, políticos y empresarios, el principio metapolítico neutro intocable que es la economía de mercado y la democracia elitista, demuestra que la transición a la democracia solo es un discurso vago o ilusorio visto bajo el prisma de una teoría política spinozista inmanente de análisis. Yendo aún más lejos, la imagen de esta forma de gobierno se parece más al de una aristocracia.

La filosofía de Spinoza y la idea de inmanencia nos permiten la crítica de la idea de democracia y el neoliberalismo. El discurso político cotidiano, aquel que se naturaliza como el verdadero, no ha sido más que la contienda por apropiarse de la idea de democracia como elemento de justificación, pero sabemos que las diferencias internas de los países que buscan autoproclamarse como democráticos en la actualidad a veces son tan marcadas, que hablar de "la" democracia como "la" mejor forma de gobierno parece un sinsentido. Y para las izquierdas más duras es casi una agresión el ser tildadas de antidemocráticas, porque ellas mismas se entienden como las detentoras por antonomasia del puritanismo democrático. Habría que hacer una revisión acabada de estas posturas. Por otro lado, Spinoza nos permite un desplazamiento del análisis. Por sobre cualquier producto institucional (parlamento, ministerios, cabildos, etc.), una democracia se define más por sus despliegues, movimientos y encuentros: la "ampliación de poder" o la "concentración de poder" como tendencias en dirección opuesta. Está forma de considerar la política tiene la virtud de ser dinámica lo que nos aleja de los personales prejuicios de lo que sea un buen o mal gobierno. La democracia es tan frágil como cualquier otra forma de organización y eso lo demuestra la historia de un país como Chile. 


\section{Referencias bibliográficas:}

Atria 2016: F. Atria. "Nueva constitución y reforma constitucional: el contenido de la forma". Anales de la Universidad de Chile (Santiago, 2016) 0 (10), n. 19-46. http://www.anales.uchile.cl/index.php/ANUC/article/ viewFile/43140/45096

Balibar 2011: É. Balibar, Spinoza y la Política (Buenos aires, 2013).

Bourdieu y Passeron 1996: P. Bourdieu y J. Passeron, La Reproducción.

Elementos para una teoría del sistema de enseñanza (Barcelona, 1996).

Bergson 2013: H. Bergson, El pensamiento y lo Moviente (Buenos Aires, 2013).

Capella 2007: J. Capella, Entrada a la Barbarie (Madrid, 2007).

Cazor 2000: K. Cazor, "Democracia y Constitución en Chile”. Rev. Derecho

(Valdivia, 2000), vol.11, pp .27-34. En:

http://mingaonline.uach.cl/pdf/revider/v11/art03.pdf

Constitución Política de la República de Chile 1980, Art. $4^{\circ}$. Consultado el 6 de septiembre de 2016. En: http://www.leychile.cl/Navegar?idNorma=17039

Cristi y Ruiz Tagle 2006: R. Cristi y P. Ruiz-Tagle, La República en Chile.

Teoría y práctica del constitucionalismo republicano (Santiago, 2006).

Delano y Translaviña 1989: M. Délano y H. Traslaviña, La Herencia de los

Chicago Boys, (Santiago, 1989).

Deleuze 2008: G. Deleuze, En Medio de Spinoza, (Buenos Aires, 2008).

Deleuze 1999: G. Deleuze, Spinoza y el Problema de Expresión, (Barcelona, 1999).

Del Pozo 2009: J. Del Pozo, Historia de América Latina y del Caribe. Desde la independencia hasta hoy, (Santiago, 2009).

Diario BBC Mundo 2005, "Chile, fin de la transición". Consultado el 5 septiembre de 2016. En:

http://news.bbc.co.uk/hi/spanish/latin_america/newsid_4682000/4682157.stm

Diario El País 2005, "En Chile la transición a la democracia no ha terminado y la reconciliación no ha comenzado. Entrevista a Jorge Edwards". Consultado el 7 de septiembre de 2016. En:

http://elpais.com/diario/2005/01/08/babelia/1105144750_850215.html

Diario La Nación 2014, “AFP al desnudo: el polémico reportaje de en la mira en 10 puntos". Consultado el 9 de septiembre de 2016. En:

http://www.lanacion.cl/noticias/economia/pension/afp-al-desnudo-elpolemico-reportaje-de-en-la-mira-en-10-puntos/2014-07-29/121730.html Espinoza 2014: L. Espinosa, "La política como física del poder en Spinoza" Res Publica: revista de historia de las ideas políticas, (Madrid, 2014), Vol. 17, No. 1, n. 33-57. En: https://revistas.ucm.es/index.php/RPUB/ article/download/45555/42843 
Gebhardt 2008: C. Gebhardt, Spinoza. (Buenos Aires, 2008).

Gueroult 1968: M. Gueroult, Spinoza, Dieu (Ethique, 1) (París, 1968).

Harvey 2007: D. Harvey, Breve Historia del Neoliberalismo (Madrid, 2007).

Hobsbawm 1994: E. Hobsbawm, Historia del Siglo XX (Buenos Aires 2008).

Jofré 1988: G. Jofre, “El Sistema de Subvenciones en Educación: la Experiencia

Chilena”. Rev. Estudios Públicos (Santiago, 1988), n. 193-237. En:

http://opech.cl/bibliografico/Doc_Financiamiento/sistema_subvenciones_ educacion_chilena.pdf

Klein 2007: N. Klein, La Doctrina del Schock. El auge del capitalismo del desastre (Madrid, 2007).

Molina, Zabala, Avetikián, Flisfisch, Godoy y Guzmán 1986: S. Molina, J. Zabala, T. Avetikián, A. Flisfisch, O. Godoy y J. Guzmán. “Acuerdo Nacional y Transición a la Democracia”. Rev. Estudios Públicos (Santiago, 1986), vol. 21, n. 1-93. En: http://www.cepchile.cl/acuerdo-nacional-ytransicion-a-la-democracia/cep/2016-03-03/183818.html

Mayol y Ahumada 2015: A. Mayol y J. M. Ahumada, Economía Política del Fracaso. La falsa modernización del modelo neoliberal (Santiago 2015).

Moulian, 2002: T. Moulian, Chile Actual. Anatomía de un mito (Santiago, 2002)

Negri 1993: T. Negri, La Anomalía Salvaje. Ensayo sobre poder y potencia en Baruch Spinoza. (Barcelona, 1993).

Salazar 2012: G. Salazar, Movimientos Sociales en Chile. Trayectoria histórica y proyección política. (Santiago, 2012).

Salazar y Pinto 1999a: G. Salazar y J. Pinto. Historia Contemporánea de Chile I: estado, legitimidad, ciudadanía (Santiago, 1999)

Salazar y Pinto, 1999b: G. Salazar y J. Pinto. Historia Contemporánea de Chile II: actores, identidad y movimiento (Santiago, 1999).

Ruiz y Boccardo 2014: C. Ruiz E y G. Boccardo. Los Chilenos Bajo el Neoliberalismo. Clases y conflicto social (Santiago, 2014).

Ruiz 2010: C. Ruiz S. De la República al Mercado. Ideas educacionales y politicas en Chile, (Santiago, 2010).

Ruiz 1993: C. Ruiz S. Seis Ensayos sobre Teoría de la Democracia (Santiago, 1993).

Spinoza 2014: B. Spinoza, Tratado Teológico-Político. (Pamplona, 2014).

Spinoza 2011: B. Spinoza, Ética. (Madrid, 2011).

Spinoza 2010: B. Spinoza, Tratado Político. (Madrid, 2010).

Spinoza 1972: B. Spinoza, Opera. (Heidelberg, 1972).

Tejeda 2015: C. Tejeda, "La importancia de la causa inmanente en la Ética de

Spinoza". Revista de filosofía, 71, n. 163-175.

https://dx.doi.org/10.4067/S0718-43602015000100013 
Urrea 2014: G. Urrea. Chile: por un Nuevo Modelo Económico. Para una sociedad democrática, solidaria y sustentable (Santiago, 2014).

Yovel 1995: Y. Yovel. Spinoza, el Marrano de la Razón (Madrid, 1995).

Virno 2003: P. Virno. Gramática de la Multitud (Madrid, 2003).

Zourabichvili 2014: F. Zourabichvili. Spinoza, Una Física del Pensamiento (Buenos Aires, 2014). 
